\title{
An Adenylate Kinase OsAK3 Involves Brassinosteroid Signaling and Grain Length in Rice (Oryza sativa L.)
}

Jiaqi Zhang ${ }^{1,2+}$, Xiuying Gao ${ }^{1,2+}$, Guang Cai ${ }^{1,2}$, Yuji Wang ${ }^{1,2}$, Jianbo Li ${ }^{1,2}$, Huaying Du ${ }^{1,2}$, Ruqin Wang ${ }^{1,2}$, Hongsheng Zhang ${ }^{1,2}$ and Ji Huang ${ }^{1,2^{*}}$ (D)

\begin{abstract}
Background: Grain size is one of the major determinants of cereal crop yield. As a class of plant polyhydroxysteroids, brassinosteroids (BRs) play essential roles in the regulation of grain size and plant architecture in rice. In a previous research, we cloned qGL3/OSPPKL1 encoding a protein phosphatase with Kelch-like repeat domains, which negatively regulates $B R$ signaling and grain length in rice.
\end{abstract}

Results: Here, we screened qGL3-interacting proteins (GIPs) via yeast two-hybrid assay and analyzed the phenotypes of the T-DNA insertion mutants of GIPs. Among these mutants, mutant osak3 presents shorter grain length and dwarfing phenotype. OSAK3 encodes an adenylate kinase, which regulates grain size by controlling cell expansion of rice spikelet glume. Overexpression of OSAK3 resulted in longer grain length. OsAK3 interacts with qGL3 in vivo and in vitro. Lamina inclination, coleoptile elongation and root inhibition experiments showed that the osak3 mutant was less sensitive to exogenous brassinolide (BL) treatment. The transcriptional level of OsAK3 was up-regulated under BL induction. In addition, RNA-Seq data indicate that OsAK3 is involved in a variety of biological processes that regulate BR signaling and grain development in rice.

Conclusions: Our study reveals a novel BR signaling component OsAK3 in the regulation of grain length, and provides novel clues for uncovering the potential functions of OsAK3 in rice growth and development.

Keywords: Grain length, Brassinosteroids, Adenylate kinase, OsAK3, qGL3

\section{Background}

As an important cereal crop, rice is widely planted all over the world. Grain weight is a major determinant of crop yield. Grain size is not only one of the decisive factors of grain weight, but also affects the appearance quality and commodity value of rice (Harberd 2015). Spikelet hull is the dominant factor to limit grain size, and its development is mainly affected by cell expansion and

\footnotetext{
*Correspondence: huangji@njau.edu.cn

†iaqi Zhang and Xiuying Gao contributed equally to this work.

1 State Key Laboratory of Crop Genetics and Germplasm Enhancement, College of Agriculture, Nanjing Agricultural University, Nanjing 210095, China

Full list of author information is available at the end of the article
}

cell proliferation ( $\mathrm{Li}$ et al. 2018). To date, many genes related to rice grain size have been identified and they involve various signaling pathways, including $G$ protein signaling, the mitogen-activated protein kinase (MAPK) signaling pathway, ubiquitin-mediated proteasome degradation pathway, transcriptional regulation and phytohormone biosynthesis or signaling pathways (Zuo and $\mathrm{Li}$ 2014; Li et al. 2018). For example, Ga protein RGA1 and $G \beta$ protein RGB1 from $G$ protein signaling pathway positively regulate rice grain size by affecting cell proliferation. Grain length increases when the GY proteins DEP1 and GGC2 bind to Gß, either alone or together. However, the Gy protein GS3 reduces grain length via interacting competitively with G $\beta$ (Fan et al. 2006; Utsunomiya et al. 
2011; Sun et al. 2018). In MAPK signaling pathway, $O$. Sativa MAPK KINASE4 (OsMKK4) interacts with and phosphorylates OsMAPK6 to control grain size (Liu et al. 2015b). Further biochemical and genetic analysis showed that O. Sativa MAPK KINASE KINASE10 (OsMKKK10), OsMKK4 and OsMAPK6 function together to control grain size (Xu et al. 2018). A major QTL locus GRAIN WIDTH2 (GW2) encodes a RING-type E3 ubiquitin ligase controlling the proliferation of spikelet hull and grain width (Song et al. 2007). The deubiquitinating enzyme WTG1/OsOTUB1 controls grain size mainly by affecting cell expansion (Huang et al. 2017). In addition, phytohormone pathways also play important roles in regulating the grain size in rice, such as O. Sativa SHORT GRAIN LENGTH (OsSGL) and BIG GRAIN3 (BG3) in cytokinin signaling (Wang et al. 2016; Xiao et al. 2019); BIG GRAIN1 (BG1), SMOS1 and Gnp4/LAX2 in auxin signaling (Aya et al. 2014; Liu et al. 2015a; Zhang et al. 2018b); GRAIN SIZE5 (GS5), SLENDER GRAIN (SLG), GLYCOGEN SYNTHASE KINASE2 (GSK2) and qGL3 in brassinosteroid (BR) signaling (Tong et al. 2012; Xu et al. 2015; Feng et al. 2016; Gao et al. 2019).

BRs are a class of steroid phytohormones that play an important role in multiple processes of plant growth and development, including cell elongation and proliferation, lamina bending, grain filling, stomatal opening, photomorphogenesis and stress responses (Clouse and Sasse 1998; Tong and Chu 2018; Li et al. 2020). In rice, when BRs present, they bind to the receptor complex O. sativa BRASSINOSTEROID-INSENSITIVE1 (OsBRI1) and O. sativa BRI1-ASSOCIATED RECEPTOR KINASE1 (OsBAK1) (Yamamuro et al. 2000; Li et al. 2009). OsBRI1 phosphorylates $O$. sativa BR-SIGNALING KINASE3 (OsBSK3) to activate BR signal, and the phosphorylated OsBSK3 increases the binding affinity for Arabidopsis bri1-SUPPRESSOR1 (AtBSU1) (Zhang et al. 2016). In Arabidopsis, BSU1 plays a positive role in BR signaling, while in rice, its homolog qGL3/OsPPKL1 plays a negative role by mediating the phosphorylation status and stability of protein kinase OsGSK3 and transcription factor (TF) O. sativa BRASSINAZOLE RESISTANT1 (OsBZR1) (Gao et al. 2019). In addition to OsBZR1, there are other TFs involving BR signaling, including DWARF AND LOW-TILLERING (DLT), SMOS1/RLA1, OsGRF4, LEAF AND TILLER ANGLE INCREASED CONTROLLER (LIC) and OVATE FAMILY PROTEIN1 (OFP1), OFP3, OFP8 and OFP19 (Tong et al. 2012; Zhang et al. 2012a; Che et al. 2015; Yang et al. 2016, 2018; Qiao et al. 2017; Xiao et al. 2017, 2020). Most of these TFs interact with and are regulated by GSK3-like kinases in BR signaling.

Adenylate Kinases (AKs; EC 2.7.4.3), also known as myokinases, a kind of highly conserved nucleoside monophosphate kinases widely existing in various organisms (Zhang et al. 2018a). This enzyme participates in maintaining nucleotide balance and energy metabolism by catalyzing the interconversion of adenine nucleotides as follows: AMP + ATP $\leftrightarrow$ ADP (Dzeja and Terzic 2009). There are nine isoenzymes of AKs described in human, and they play a central role in different intracellular compartments (Panayiotou et al. 2014). AK1, AK5, AK7 and AK8 were located in cytosol (Panayiotou et al. 2011). AK2, AK3 and AK4 are mitochondrial isoenzymes, but AK2 was found in the mitochondrial intermembrane space, and the other two isoenzymes were identified in the mitochondrial matrix (Noma et al. 2001). In addition, AK3 is a GTP: AMP phosphotransferase. Unlike other enzymes, its substrate is not ATP, but GTP. AK6 is located in nucleus (Ren et al. 2005), and AK9 is located in both cytoplasm and nucleus (Amiri et al. 2013). In Arabidopsis thaliana, ADENYLATE KINASE 6 (AAK6) was identified as an orthologue of human AK6 isoform AD-004 and it's also located in the nucleus. The aak6 mutant repressed stem growth compared with wild-type plants, indicating that AAK6 is an essential stem growth factor (Feng et al. 2012). The loss-of-function of $A A K 6$ leads to the accumulation of $80 \mathrm{~S}$ ribosomes, thereby affects the cell proliferation and cell size homeostasis in Arabidopsis root growth (Slovak et al. 2020). The absence of Arabidopsis PLASTIDIAL ADENYLATE KINASE 1 (AtPADK1, also known as $A M K 1$ ) increases the photosynthetic amino acid biosynthesis and promotes plant growth (Carrari et al. 2005). Analysis of co-response in Arabidopsis showed that ADENOSINE MONOPHOSPHATE KINASE 2 (AMK2) and AMK5 expression levels are positively correlated with the expression of photosynthesis and major carbohydrate metabolism genes, while $A M K 3, A M K 4$ and $A M K 1$ are negatively correlated with photosynthesis genes and not related to carbohydrate metabolism genes (Lange et al. 2008). OsAK1 is the closest homolog of Arabidopsis AMK2. OsAK1 located in chloroplast and silencing OsAK1 in Epi-ak1 led to a phenotype with albinism of young leaves and panicles along with abnormal chloroplast structure (Wei et al. 2017).

To understand the qGL3-mediated BR signaling, we performed a yeast two-hybrid ( $\mathrm{Y} 2 \mathrm{H})$ assay to screen qGL3-interacting proteins (GIPs). Among these T-DNA insertion mutants of GIPs, osak3 exhibited the BR repressed phenotype. In this work, we revealed that OsAK3, encoding an adenylate kinase (Kawai et al. 1992), is a novel regulator of rice BR signaling.

\section{Results}

\section{OsAK3 Physically Interacts with qGL3}

We screened the qGL3-interacting proteins via yeast two-hybrid and identify a rice adenylate kinase (OsAK3) 
for further analysis (Fig. 1a). Compared with qGL3 from the rice cultivar 9311, qGL3 from the N411 variety is unable to dephosphorylate OsGSK3 due to amino acid changes in its Kelch domain (D364E) (Zhang et al. 2012b; Gao et al. 2019). Therefore, we analyzed whether the amino acids mutation of qGL3 affects its interaction with OsAK3. Our results showed that both qGL3 $3^{9311}$ and qGL3 $^{\mathrm{N} 411}$ interact with OsAK3 in yeast cells (Fig. 1a).

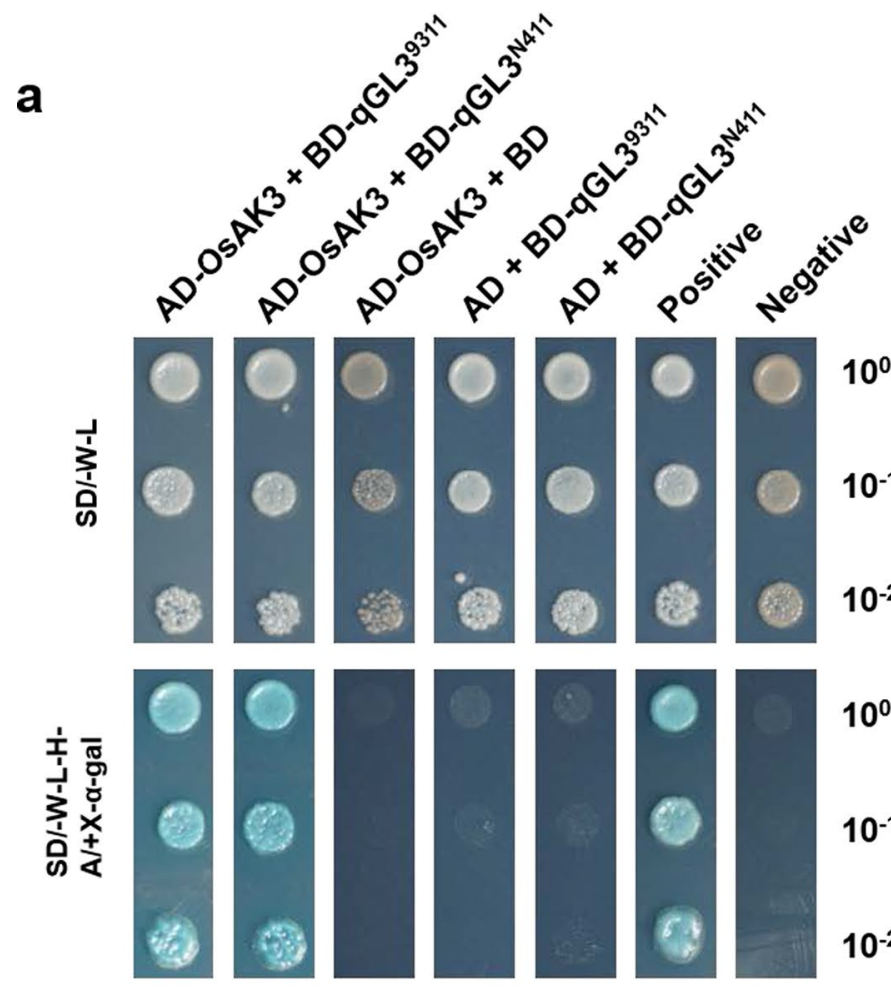

b

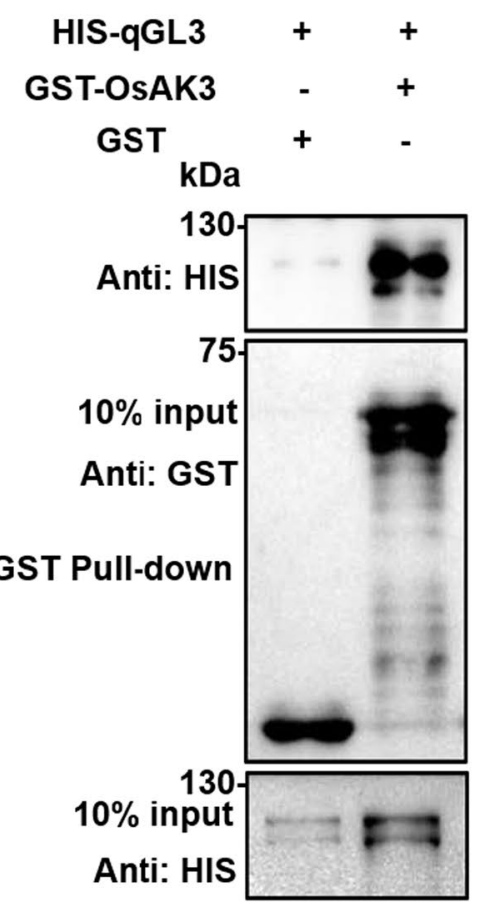

C
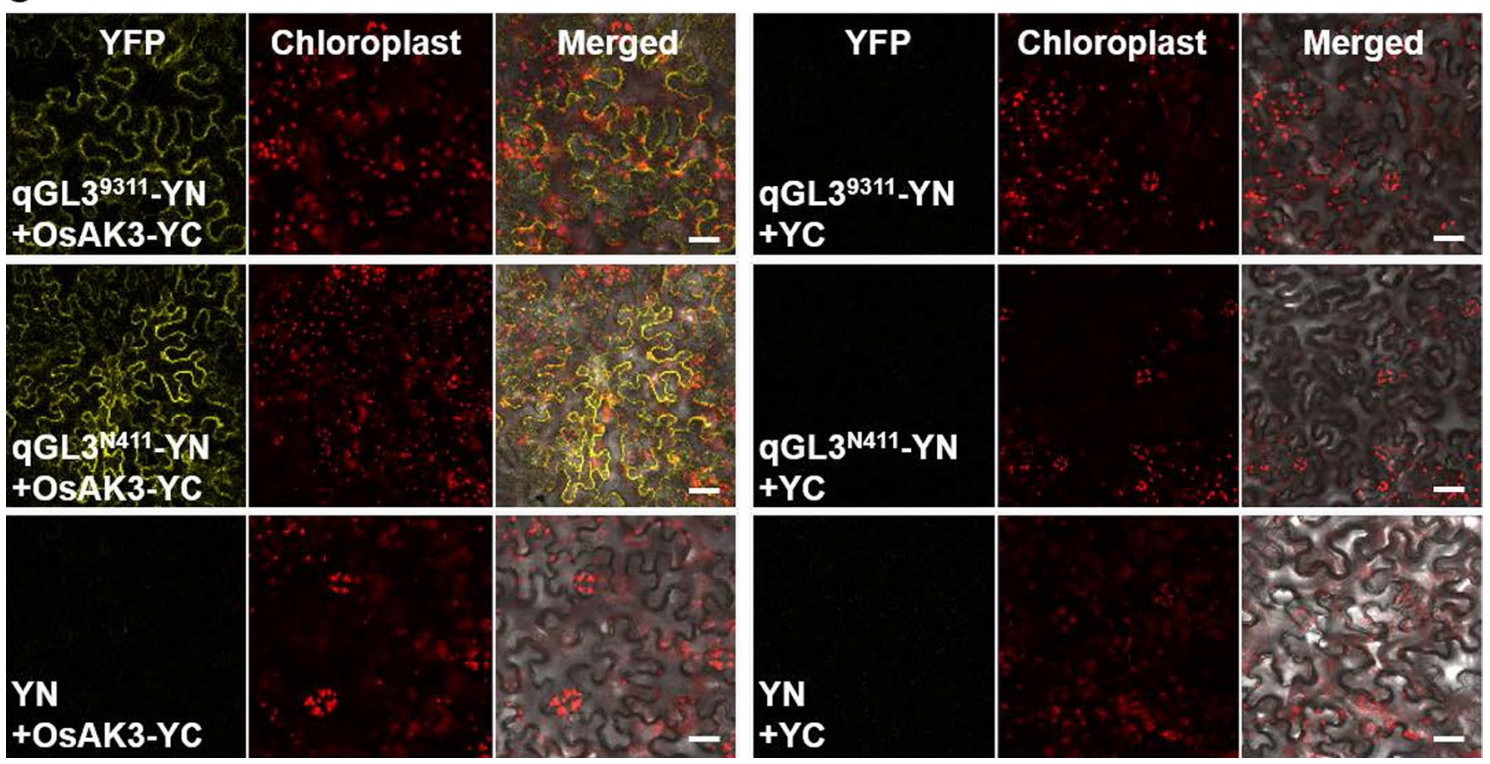

Fig. 1 OsAK3 physically interacts with qGL3. a Interaction between OSAK3 and qGL3 in Y2H analysis. $\mathbf{b}$ Interaction between OsAK3 and qGL3 in GST pull-down assay. Immunoblots were probed with anti-GST and anti-His respectively. c BiFC analysis of the interaction between OsAK3 and qGL3 (scale bars, $50 \mu \mathrm{m}$ ) 
Glutathione S-transferase (GST) pull down assay was used to study the in vitro interaction between OsAK3 and qGL3. OsAK3 and qGL3 were separately fused with GST- or His- tag to express soluble recombinant proteins in Escherichia coli. The western blot showed that GSTOsAK3 strongly bound to His-qGL3 protein (Fig. 1b).

The bimolecular fluorescence complementation (BiFC) was employed for further confirming the in vivo interaction between OsAK3 and qGL3. OsAK3 was fused to the C-terminal fragment of yellow fluorescent protein (YFP) to construct OsAK3-YC, and qGL3 was fused to the $\mathrm{N}$-terminal fragment of YFP to construct qGL3 ${ }^{9311}-\mathrm{YN}$ or qGL3 ${ }^{\mathrm{N} 411}-\mathrm{YN}$. OsAK3-YC and qGL3 ${ }^{9311}$-YN/qGL3 ${ }^{\mathrm{N} 411}$ YN were co-expressed in Nicotiana benthamiana leaves, and the strong YFP fluorescence was observed in the cytoplasm. The empty vector $\mathrm{YN}$ or $\mathrm{YC}$ co-expressed with OsAK3-YC or qGL3 ${ }^{9311}-\mathrm{YN} / \mathrm{qGL} 3^{\mathrm{N} 411}-\mathrm{YN}$ was used as negative controls (Fig. 1c). These experiments demonstrated that OsAK3 physically interacts with qGL3 in vivo and in vitro.

\section{Gene Expression, Subcellular Localization and Kinase Activity of OsAK3}

The quantitative real-time PCR (RT-qPCR) was performed to characterize the expression patterns of $O s A K 3$ in rice. The results revealed that OsAK3 gene shows highest expression level in the seedling roots and young panicles (Fig. 2a). To investigate the subcellular

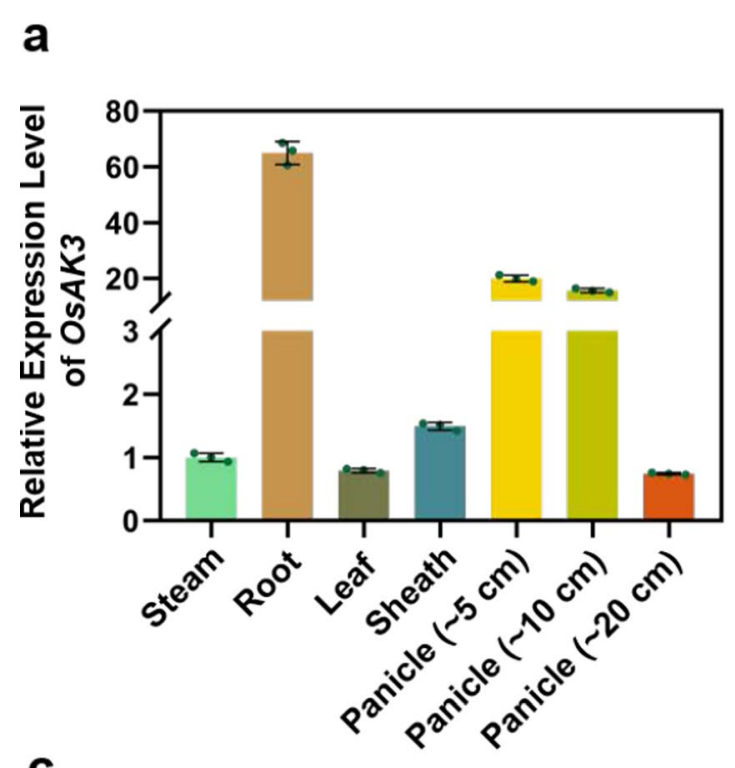

C

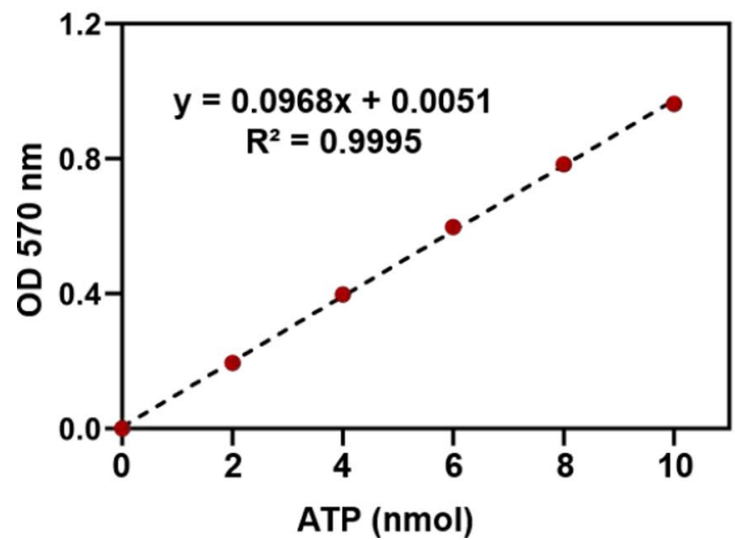

b
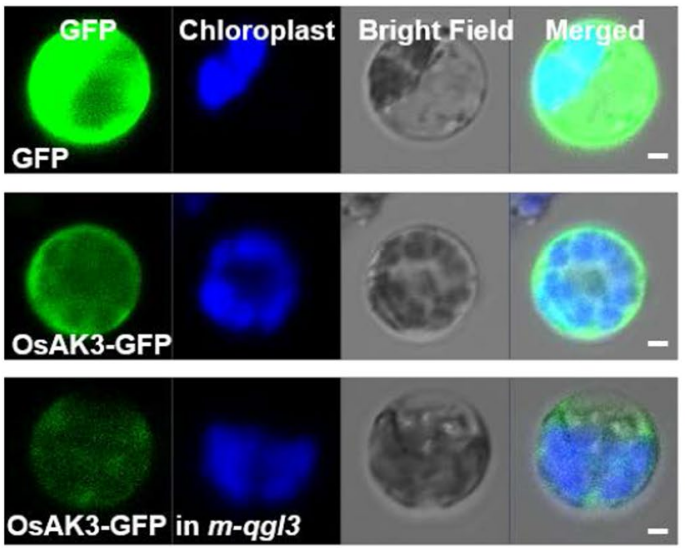

d

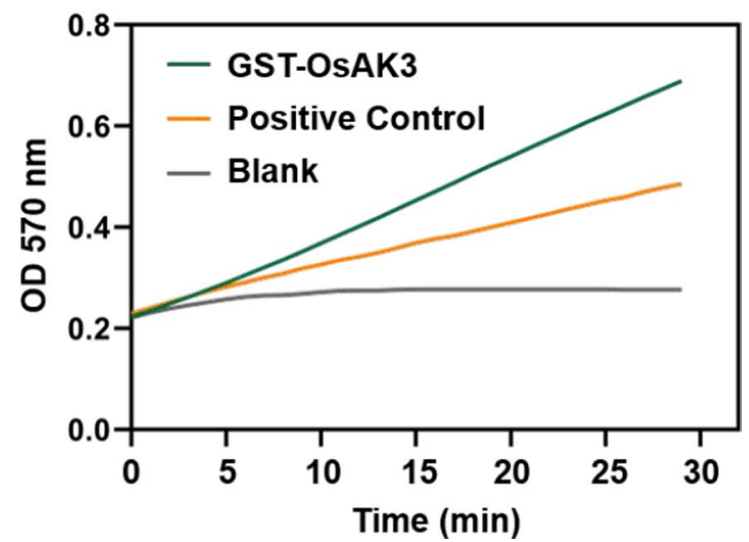

Fig. 2 OsAK3 is a functional adenylate kinase localized in cytoplasm. a RT-qPCR analysis of OsAK3 expression in various rice tissues. b Subcellular localization of OsAK3 in rice protoplast. m-9g/3 mutant was used to observe the effect of the absence of qGL3 on OsAK3 localization (scale bar, $2 \mu \mathrm{m})$. DJ was used as a wild type. c ATP Standard Curve. $\mathbf{d}$ Colorimetric quantitation of Adenylate Kinase Activity in GST-OsAK3 (0.5 ng) and Positive Control $(5 \mu \mathrm{l})$ 
localization of OsAK3, we fused OsAK3 to the N-terminus of the green fluorescent protein (GFP). GFP and OsAK3-GFP were transiently expressed in rice protoplasts using PEG-mediated transformation, respectively. OsAK3-GFP signals was observed throughout the cytoplasm (Fig. 2b).

Adenylate kinase activity assay was performed to kinetically measure adenylate kinase activity by detecting adenosine triphosphate (ATP) level, which generated from adenosine diphosphate (ADP). As shown in Fig. 2c, d, OsAK3 has high-level adenylate kinase activity in vitro. According to the ATP production of 14-28 min, the catalytic activity of OsAK3 was calculated to be $341.94 \mathrm{mU} / \mathrm{ug}$. We also examined OsAK3 activity in the presence of qGL3 in vitro. When qGL3 was added, the reduction level of OsAK3 catalytic activity was $19.63 \mathrm{mU} / \mathrm{ug}$. Based on this analysis, we think that qGL3 likely has no significant effect on OsAK3 activity in vitro (Additional file 2: Fig. S1).

\section{The Homologue OsAK4 also Interacts with qGL3}

OsAK3 encodes an adenylate kinase and has 73.7\% homologous with OsAK4 (Os11g0312220) in nucleotide sequences and $90.8 \%$ homologous with OsAK4 in amino acid sequences (Kawai et al. 1992). As previously stated, there are nine adenylate kinase isoenzymes in human body, which are mainly divided into three categories according to their distribution location (Ionescu 2019). Arabidopsis thaliana AAK6 has been reported to have the highest homology to human $A K 6$, both localized in the nucleus (Slovak et al. 2020). OsAK1 and Arabidopsis thaliana AMK2 present closely homologous, both of them are located in chloroplasts and related to photosynthesis (Wei et al. 2017). These results are consistent with our phylogenetic analysis (Additional file 2: Fig. S2a). Arabidopsis thaliana $A M K 3$ and $A M K 4$ are the closest homologs to human $A K 2$, which is highly expressed in the mitochondrial intermembrane space. However, $A M K 3$ and $A M K 4$ were predicted to have no signal target mitochondrial or chloroplast transit peptide (Lange et al. 2008). The phylogenetic analysis showed that OsAK3 and OsAK4 belong to the same branch with Arabidopsis thaliana $A M K 3$ and $A M K 4$ (Additional file 2: Fig. S2a). Therefore, we also verified whether OsAK4 interacts with qGL3. Y2H analysis showed that OsAK4 also interacts with qGL3 (Additional file 2: Fig. S2b). Similarly, BiFC assay demonstrated that OsAK4 interacts with qGL3 in cytoplasm (Additional file 2: Fig. S2c). These results suggest that adenylate kinases interacting with qGL3 may function redundantly to affect rice growth and development.

\section{Mutation of OsAK3 Causes Small Grains and Dwarfism}

To explore whether OsAK3 is involved in grain length and BR signaling, we identified a T-DNA insertion mutant osak3 (Os12g0236400, PFG_3A-01370.L) with a smaller grain size, shorter plant height, and reduced tillers (Fig. 3a, c, d). Compared with wild-type Dongjin (DJ), the average of grain length, width and 1000-grain weight in the osak3 mutant were significantly decreased (Fig. 3a, $\mathrm{e}-\mathrm{g})$. We confirmed the insertion site and the reverse transcription semi-quantitative PCR (RT-sqPCR) identification indicated that the OsAK3 expression was affected (Additional file 2: Fig. S3a-c). To confirm the effect of the OsAK3 mutation on rice phenotype, we obtained another line of the T-DNA mutant, osak3-r (PFG_3A-01370.R, Additional file 2: Fig. S3d-f) from the rice mutant library. The osak3-r also showed a dwarfism phenotype with reduced grain length and width and decreased 1000grain weight (Additional file 2: Fig S4a, c-g). Since both the size and number of spikelet glume cells affect the final size of the seed (Li et al. 2018), we used scanning electron microscopy (SEM) to examine the glume cells of osak3, osak3-r and DJ. We found that the average cell length and width of outer glume in osak3 and osak3-r mutants was significantly decreased compared to DJ, and the cell number was remarkably increased (Fig. 3b, h; Additional file 2: Fig. S3g, h, S4b, $h-j)$. These results indicate that OsAK3 modulates rice grain size by controlling cell expansion.

\section{Altered BL Sensitivity in osak3 Mutants}

Given the presence of BR-deficient phenotypes such as dwarfism, reduced grain length, shorter internodes and increased number of internodes (Fig. 3a; Additional file 2: Fig. S4a), we hypothesized that OsAK3 is involved in the rice $B R$ pathway. To explore the effect of $B R$ signaling on OsAK3 expression, DJ was treated with exogenous $\mathrm{BL}$ and sampled in time periods for RT-qPCR analysis. These results showed that $\mathrm{BL}$ treatment maintained OsAK3 expression around a twofold level within 6 h (Fig. 4a), indicating that $\mathrm{BL}$ induces OsAK3 expression. To further investigate the relationship between OsAK3 and BR signaling, we examined the sensitivity of OsAK3 transgenic plants subjected to BL treatment. The coleoptile elongation assay showed that osak 3 mutant had a markedly shorter coleoptile elongation than DJ (Fig. 4b, c). For root elongation assay, as expected, osak 3 was insensitive to $\mathrm{BL}$ (Fig. 4b, d). Although the coleoptile of mutant osak3-r did not show the same insensitive phenotype to BL treatment, but its root elongation was significantly suppressed compared with DJ (Additional file 2: Fig S5a-c).

We also performed BL-induced lamina inclination experiments. The lamina joint bending of osak3 mutant 


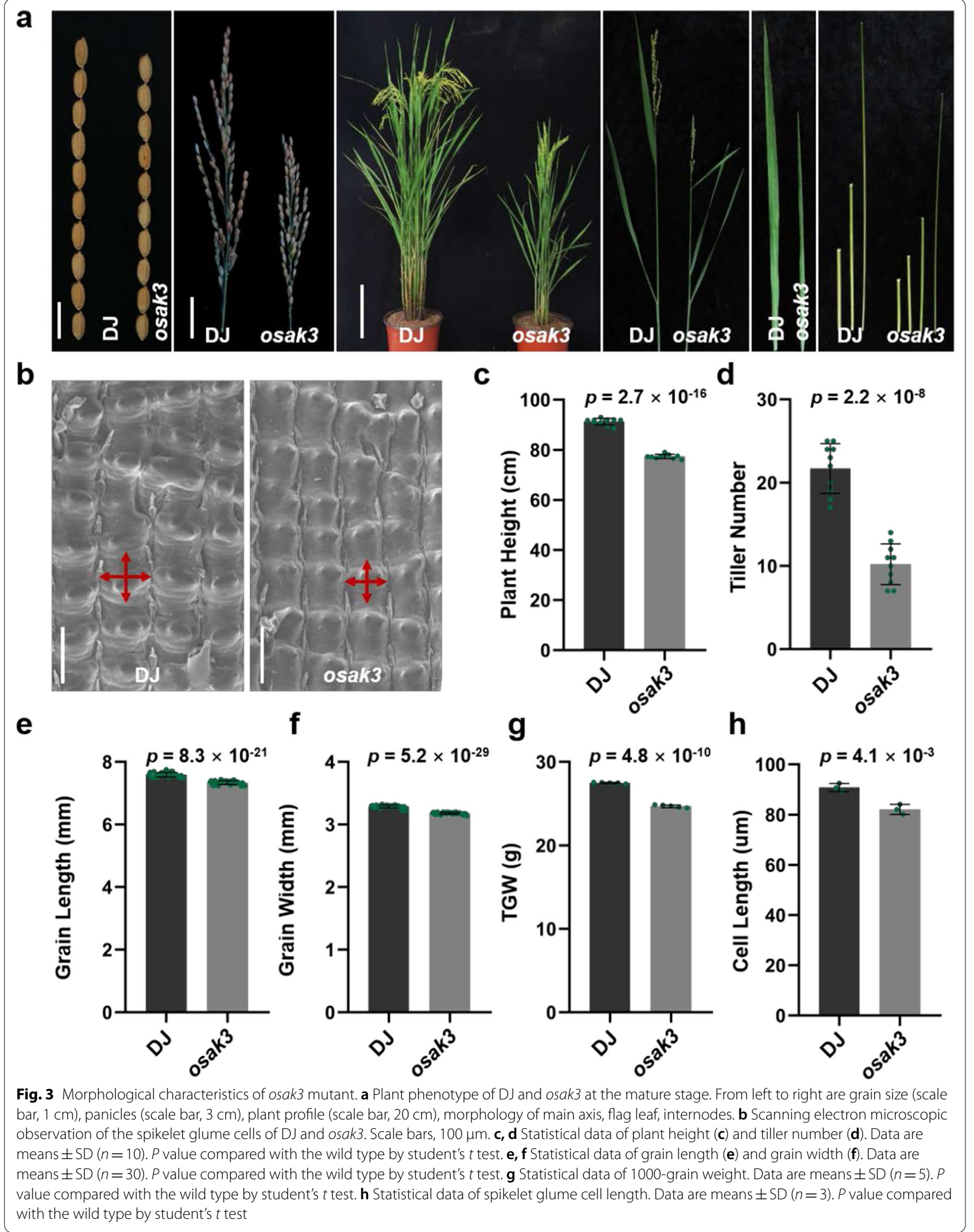


a

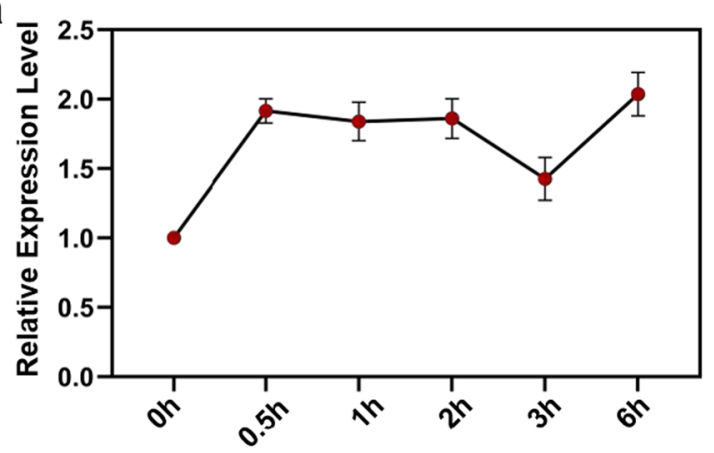

C

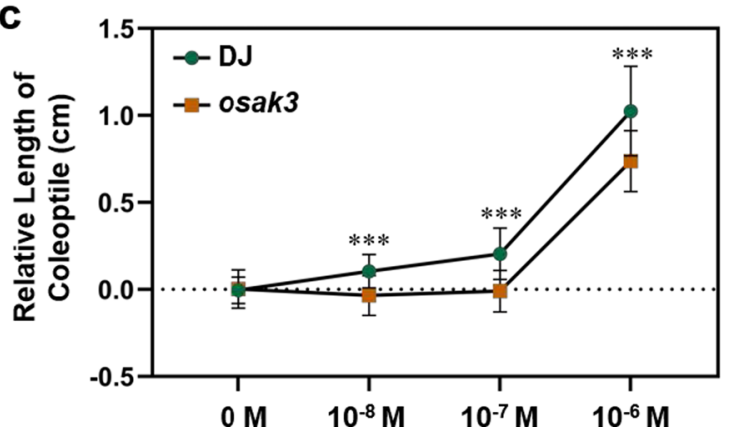

b
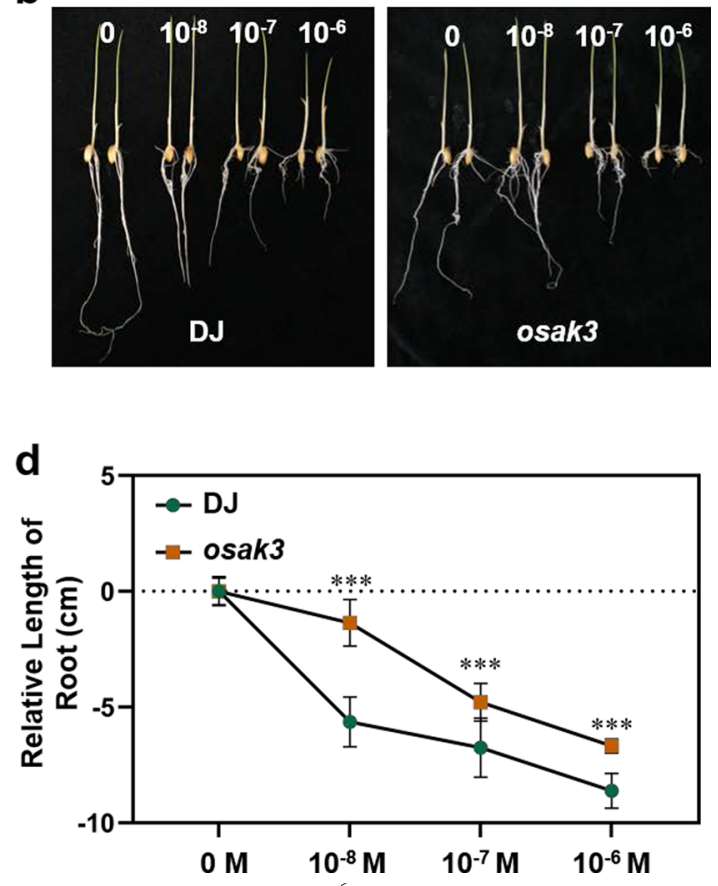

Fig. 4 OsAK3 is involved in rice brassinosteroid signaling. a RT-qPCR analysis of OsAK3 expression under $10^{-6} \mathrm{M} B L$ treatment. Data are means $\pm S D$ $(n=3)$. b Coleoptile elongation analysis and root inhibition analysis of DJ and osak3 mutant in response to $0 \mathrm{M}^{1} 10^{-8} \mathrm{M}, 10^{-7} \mathrm{M}$ and $10^{-6} \mathrm{M} \mathrm{BL} . \mathbf{c}$ Statistical data of coleoptile elongation analysis in DJ and osak3 mutant. The data was analyzed with the relative coleoptile length between $\mathrm{BL}$ treatment and mock. Data are means \pm SD $(n=20)$. Statistical analyses were performed by student's $t$ test. ${ }^{* * *} P$ value $<0.001$. $\mathbf{d}$ Statistical data of root inhibition analysis in DJ and osak3 mutant. The data was analyzed with the relative root length between BL treatment and mock. Data are means \pm SD $(n=20)$. Statistical analyses were performed by student's $t$ test. ${ }^{* *} P$ value $<0.001$

showed no obvious change compared with DJ under mock treatment. After incubation in $10^{-6} \mathrm{M}$ BL for 3 days, the lamina joint bending of DJ reached approximately $86^{\circ}$, while that of osak3 mutant was only about $53^{\circ}$ (Additional file 2: Fig. S6a, b), revealing that the mutation of OsAK3 showed less sensitivity under BL treatment. We also detected endogenous castasterone (CS) level in osak3 and DJ, and found that CS content was significantly reduced in the mutant (Additional file 2: Fig. S6c).

\section{Overexpression of OsAK3 Increased Grain Length}

To further validate the function of $O s A K 3$ in grain development, we constructed an overexpression vector that utilizes the Cauliflower Mosaic Virus 35S promoter to drive the expression of OsAK3 cDNA. This vector was then transformed into the callus of wild-type Nipponbare (NIP) using Agrobacterium tumefaciens-mediated transformation and two transgenic lines were obtained. RTqPCR analysis showed that the transcript level of OsAK3 in OsAK3-OX9 and OX11 remarkably increased compared to that in NIP (Fig. 5b). Grain length increased significantly and plant height were weakly affected in these two lines compared with NIP (Fig. 5a, c, d). Interestingly, when we performed BL sensitivity analysis on OsAK3 overexpressing materials, it was found that no significant changes in the coleoptile and root length of OsAK3-OXs compared with NIP before and after BL treatment (Additional file 2: Fig. S7a-d).

\section{Expression of BR Biosynthetic Genes were Affected by OsAK3}

We then examined the expression levels of several BRrelated genes in OsAK3-related transgenic plants by RT-qPCR (Fig. 6a, b). The expression of BR biosynthetic gene, $O s D 2$, was upregulated in osak3, but the expression of both OsDWF and OsDWF4 was reduced. The BR signaling related gene OsBZR1 was also down-regulated in osak3. Western blot showed that the protein level of OsBZR1 also decreased in osak3 (Fig. 6c). OsBZR1 can directly bind to the promoter region of OsILI1 to activate its expression (Zhang et al. 2009). In osak3, the downregulation of OsBZR1 expression was followed by a lower OsILI1 expression (Fig. 6a). The BR synthesis genes were mainly up-expressed in the overexpressed materials (Fig. 6b), and OsBZR1 showed no obvious change in OsAK3-OX9 plants (Fig. 6b, d). 

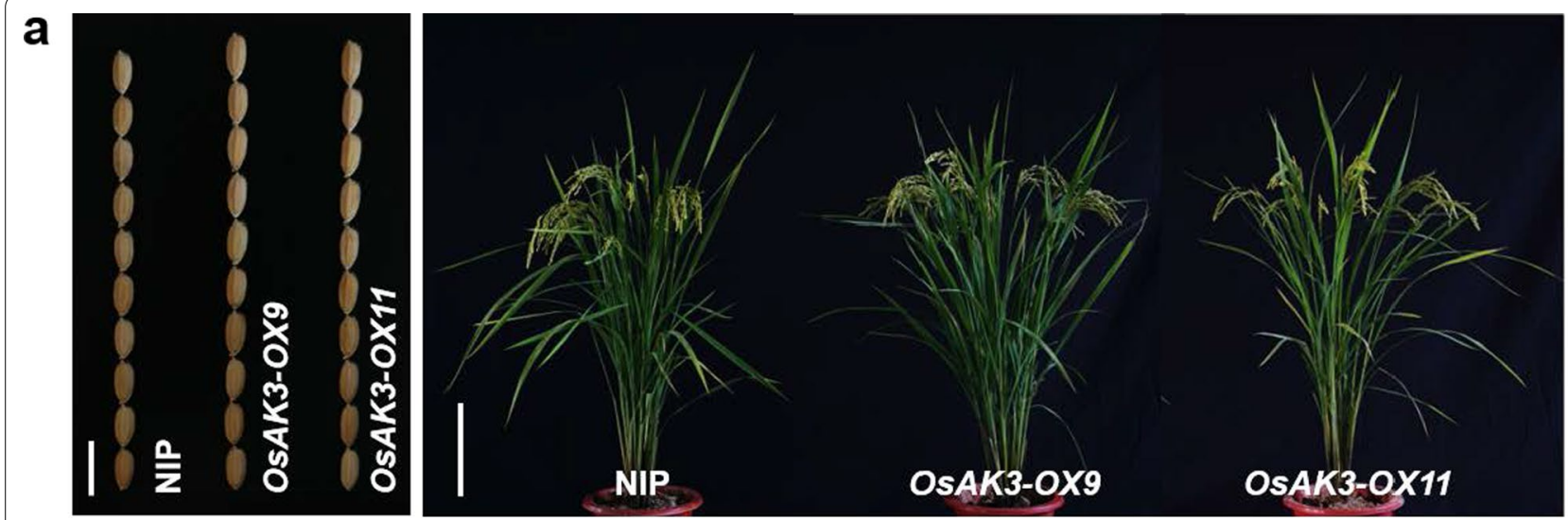

b

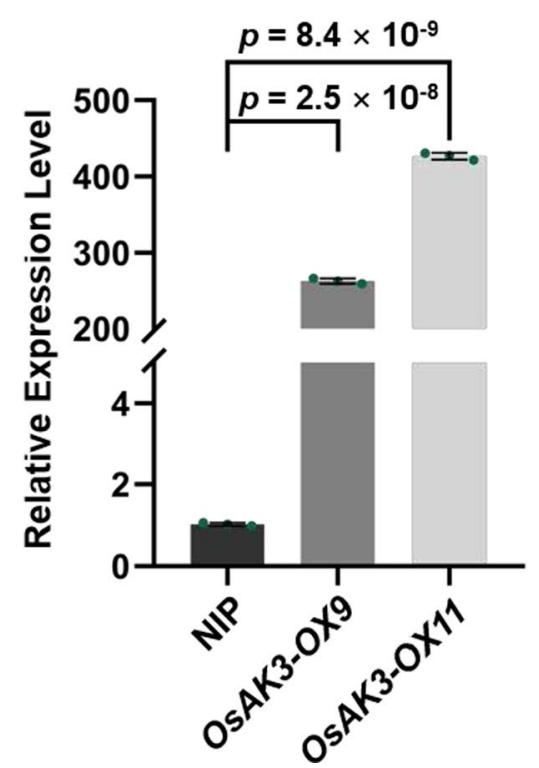

C

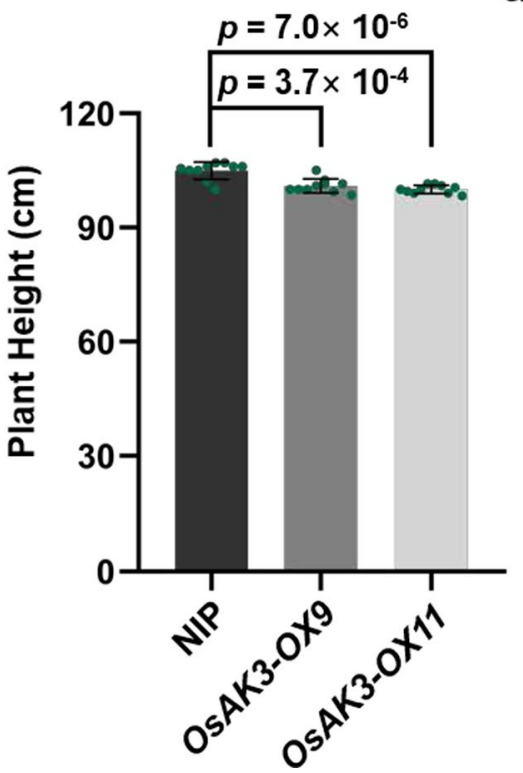

d

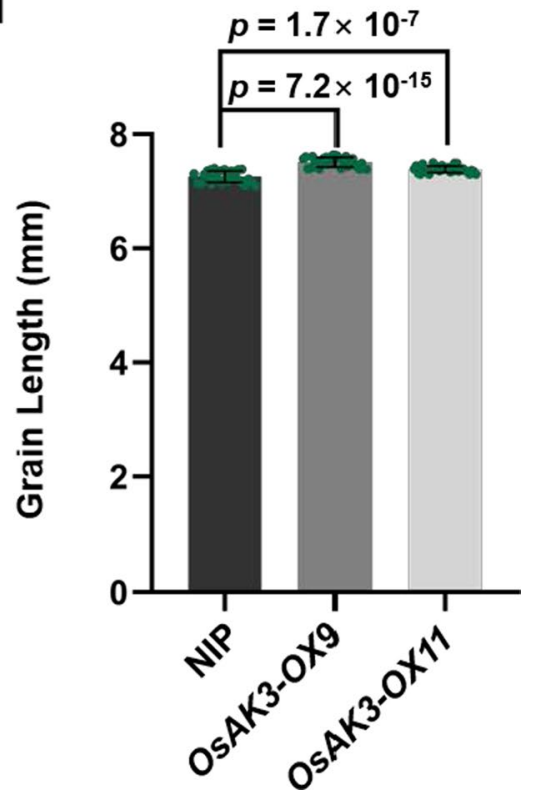

Fig. 5 Overexpression of OsAK3 increased grain length. a Plant phenotype of NIP, OsAK3-OX9 and OsAK3-OX11 at the mature stage. From left to right are grain size (scale bar, $1 \mathrm{~cm}$ ) and plant profile (scale bar, $20 \mathrm{~cm}$ ). b RT-qPCR analysis of OsAK3 expression in two overexpression lines. Data are means \pm SD $(n=3)$. $P$ value compared with the wild type by student's $t$ test. c Statistical data of plant height. Data are means \pm SD $(n=10)$. $P$ value compared with the wild type by student's $t$ test. $\mathbf{d}$ Statistical data of grain length. Data are means \pm SD $(n=30)$. $P$ value compared with the wild type by student's $t$ test

Transcriptome Analysis of the osak3 Mutant by RNA-seq To further investigate how OsAK3 functions in rice growth and development, we performed RNA-seq analysis using the young panicles $(\sim 10 \mathrm{~cm})$ of DJ and osak3 mutant. An average of $\sim 43.6$ million clean reads per sample was attained from DJ and osak3 young panicles cDNA libraries. Cluster analysis of all differentially expressed genes (DEGs) is shown in Fig. 7a. A total of 2843 genes were shown to be differentially expressed between the two genotypes (Fold change $>2, P$ value $<0.05$ ), including 1280 upregulated and 1563 downregulated genes (Fig. 7b). We selected 20 genes for RT-qPCR assay to verify the transcriptomic sequencing results. The expression levels of these 20 genes were consistent with the RNA-seq results, indicating the high quality of the transcriptomic data (Additional file 2: Fig. S8a, b; Additional file 3: Table S2). Gene Ontology (GO) enrichment analysis were performed on all DEGs, and the top 10 terms with the highest significance in biological process (BP), molecular function (MF), and cell component (CC) category were shown in the Fig. 7c. In biological process category, diterpenoid metabolic process, lipid metabolic process, and carbohydrate metabolic process had the smallest FDR (Fig. 7c). Enrichment of DEGs in these 
a

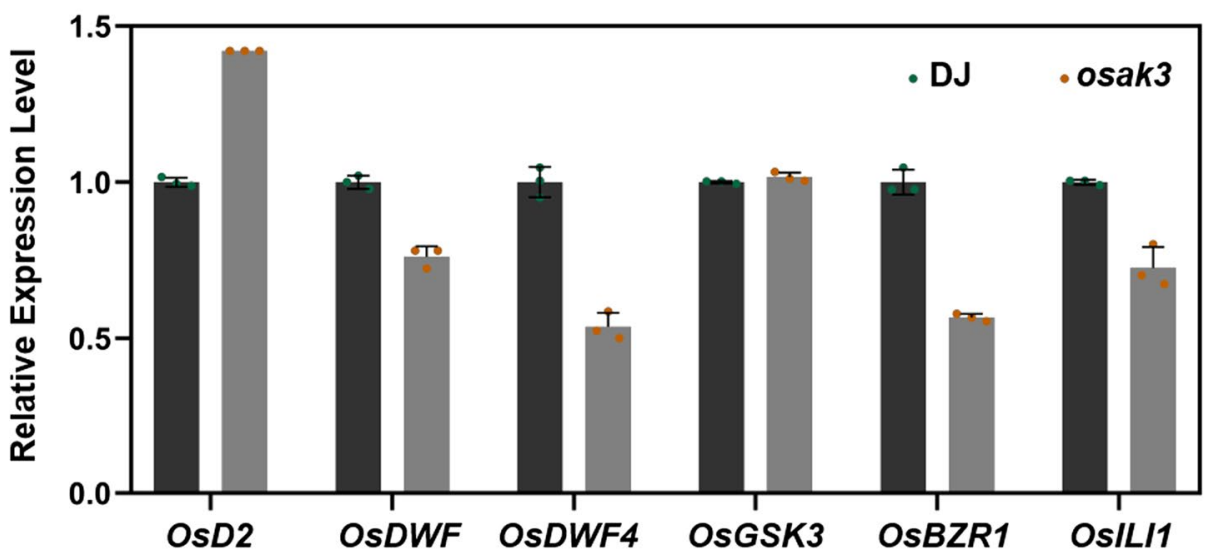

b

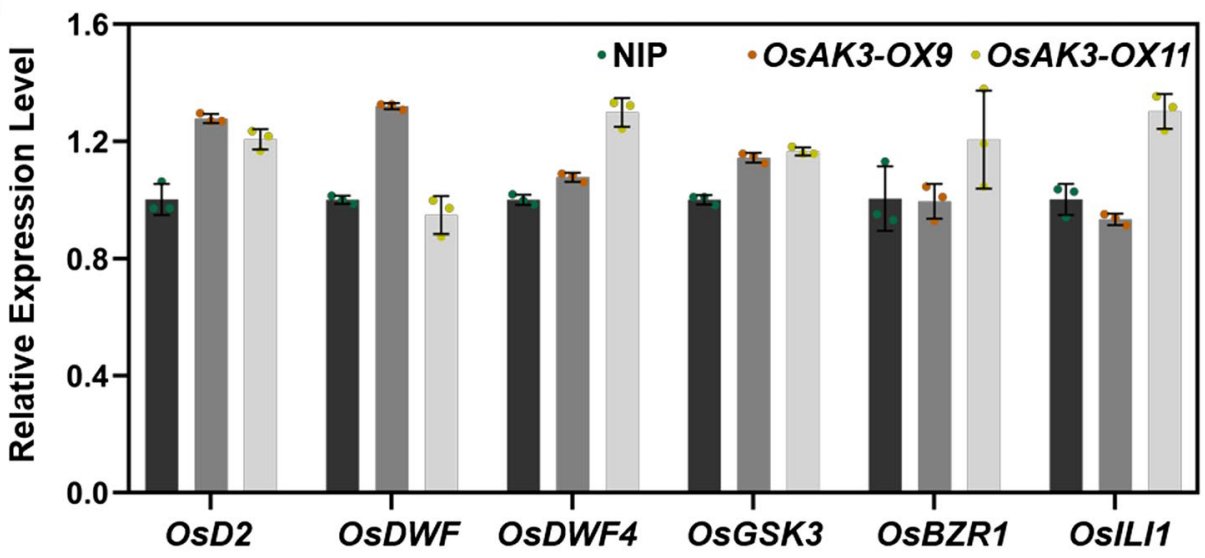

C

d
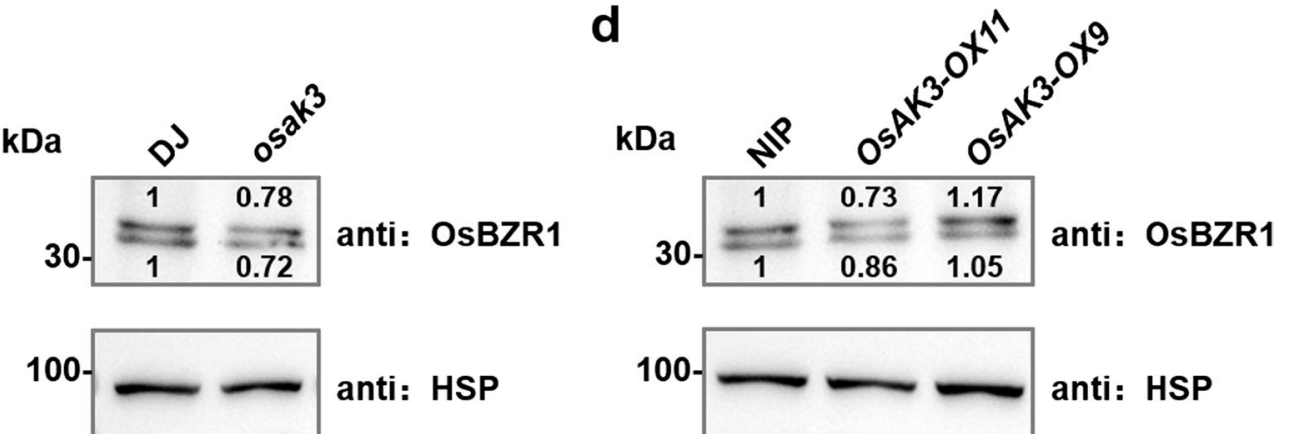

anti: HSP

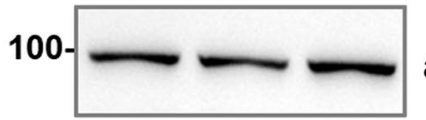

anti: HSP

Fig. 6 Expression patterns of BR biosynthesis and signaling-related genes were altered in osak3. a RT-qPCR analysis of OsD2, OsDWF, OsDWF4, OsGSK3, OsBZR1 and OsILI1 expression in DJ and osak3. Data are means $\pm S D(n=3)$. b RT-qPCR analysis of OsD2, OsDWF, OsDWF4, OsGSK3, OsBZR1 and OsILI1 expression in NIP and OSAK3-OXs plants. Data are means \pm SD $(n=3)$. c Western-blot analyses of OsBZR1 protein level in DJ and osak3. d Western-blot analyses of OsBZR1 protein level in NIP and OsAK3-OXs plants

processes indicate that OsAK3 may be involved in regulating lipid and starch metabolic, thus affecting rice grain quality. In molecular function category, DEGs are mainly related to hydrolase activity, hydrolyzing O-glycosyl compounds, and hydrolase activity, acting on glycosyl bonds (Fig. 7c). This suggests that OsAK3 may affect the function of certain proteins by mediating their glycosylation modifications (Gachon et al. 2005). In cell component category, the most significant GO terms were related to vesicles (Fig. 7c).

In particular, the genes involving BR signaling and grain size were analyzed. The rice positive grain size regulators $O s S G L$ and PGL1 were down-regulated in osak3, while the expression levels of GS2, GW8 and 
a

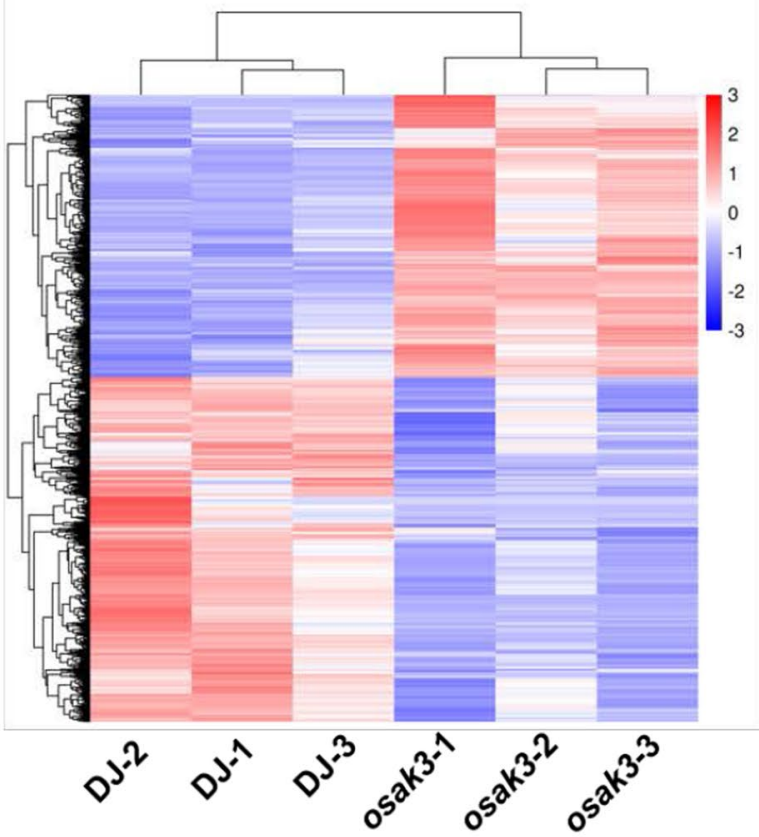

b

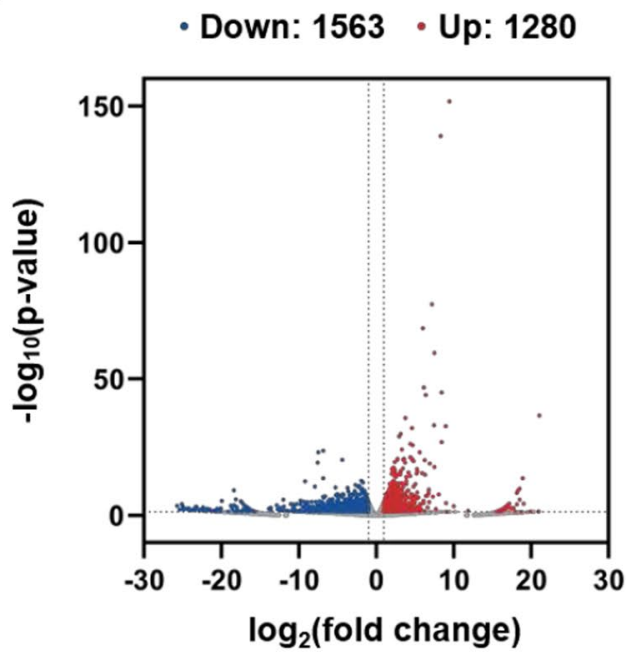

C

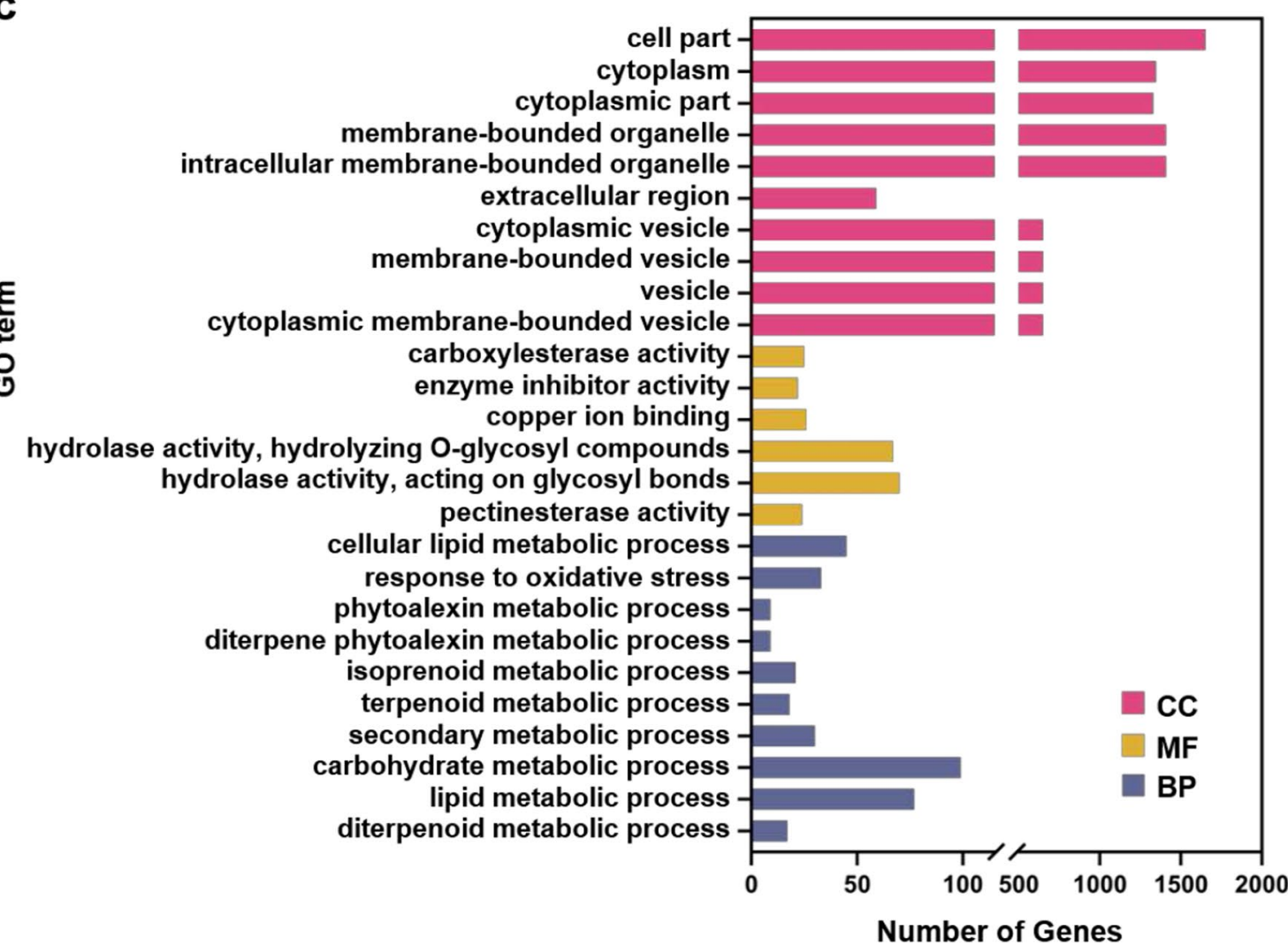

Fig. 7 RNA-seq shows that OSAK3 is involved in multiple metabolic pathways. a Hierarchical clustering of the DEGs between DJ and osak3 mutant plants. Each line with three biological replicates. DEGs were defined by absolute log2 Fold Change $>1$ and $P$ value $<0.05$. Scale bar shows fold changes, values are normalized by z-score scheme, red and blue color indicate up- and down-regulated, respectively. b Volcano map of DEGs between DJ and osak3. c Significantly enriched GO terms of DEGs between DJ and osak3. GO terms were sorted based on FDR-adjusted $P$ value $<0.05$ 
$S L G$ were increased. Similarly, the negative regulator of rice grain size, $S G 1$, was up-regulated in osak3, but the transcript level of OsFWL3 was decreased (Fig. 8a, b). This indicates that the mutation of OsAK3 affected the expression of the grain length and BR response genes. The pathway analysis of DEGs using Mapman software revealed that, in addition to their roles in the BR signaling pathway, DEGs were enriched in other hormone signaling pathways such as auxin, abscisic acid, gibberellin and ethylene (Additional file 2: Fig. S9a-d). We screened genes related to the aforementioned hormone

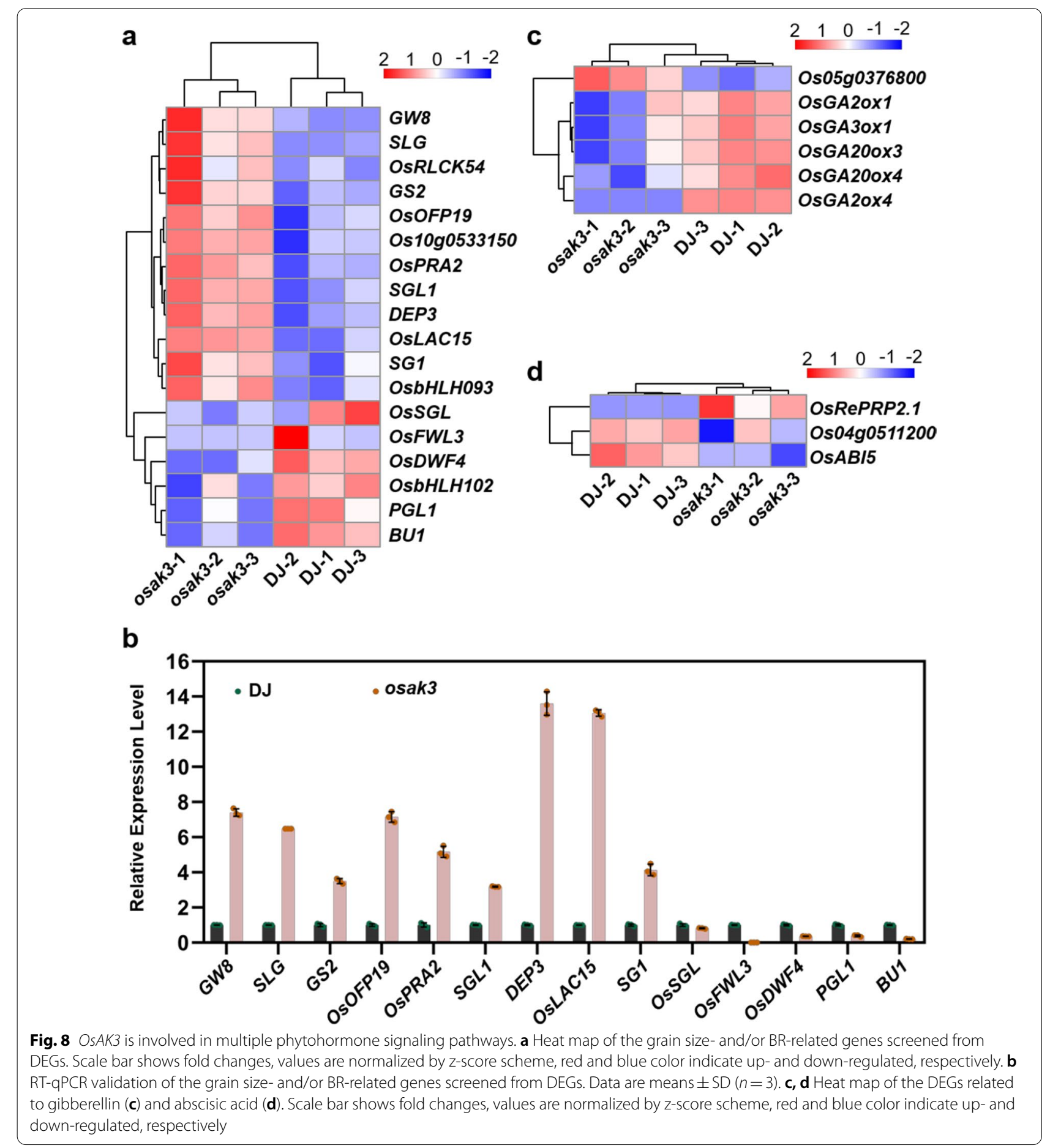


pathways from DEGs for the heat map analysis (Fig. 8c, $\mathrm{d}$ and Additional file 2: Fig. S10a-c).

\section{Discussion \\ OsAK3 Regulates Rice Grain Length and BR Signaling}

There are various signaling pathways controlling rice grain size (Zuo and Li 2014; Li et al. 2018). In this study, we obtained two T-DNA insertion mutants osak3 and osak3-r with reduced grain size and decreased plant height, implying the roles of OsAK3 in the regulation of grain length (Fig. 3a, c, e, f; Additional file 2: Fig. S4a, c, e, f). The mutation of OsAK3 could shorten the length and narrow the width of mutant osak3 and osak3-r glume cells compared with the wild type DJ (Fig. 3b, h; Additional file 2: Fig. S3g, h, S4b, h-j). Therefore, suggesting that OsAK3 controls rice grain size by regulating cell expansion. Since OsAK3 interacts with qGL3 and qGL3 is a critical modulator in BR signaling pathway, OsAK3 regulates grain length probably through regulating $B R$ signaling.

Therefore, the BL sensitivity assay was performed to study the involvement of OsAK3 in BR signaling. As expected, loss-of-function of OsAK3 displayed a BRinsensitive phenotype. Above all, lamina joint inclination, coleoptile elongation and root growth inhibition assays indicated that osak3 was less sensitive to exogenous BL treatment (Fig. 4b-d; Additional file 2: Fig. S6a, b). Interestingly, treatment of exogenous BL had no impact on OsAK3-OXs plants (Additional file 2: Fig. S7). We hypothesized that different expression level of OsAK3 has different effects on BR signaling, since OsAK3 catalyzes a reversible trans-phosphorylation in plant cells. Besides, BL treatment maintains OsAK3 expression around a twofold higher level within $6 \mathrm{~h}$ (Fig. 4a), indicating that BR induces OsAK3 transcription. Furthermore, the protein level of OsBZR1 decreased in osak3 mutant compared with wild type. Interestingly, the protein level of OsBZR1 was also down regulated in OsAK3-OX11, we speculate that there is a dosage effect due to the higher expression of OsAK3 in OsAK3-OX11 compared to OsAK3-OX9, which exerts a negative feedback regulation on the protein levels of OsBZR1. Collectively, OsAK3 is a novel component in rice $\mathrm{BR}$ response.

\section{OsAK3 Physically Interacts with qGL3}

Towards an investigation of the molecular mechanism of OsAK3 in the BR-regulated grain development, the $\mathrm{Y} 2 \mathrm{H}$, GST pull-down and BiFC assays were employed to detect the interaction between OsAK3 and qGL3 (Fig. 1a-c). Although OsAK3 and qGL3 interact with each other, but the alterations in qGL3 phosphatase activity do not affect the interaction, and the absence of qGL3 does not affect the localization of OsAK3 in the cytoplasm (Fig. 2b).
qGL3 acts as a protein phosphatase that dephosphorylates OsGSK3 to function in regulating rice grain length. In addition, it has been reported that human AK2 can directly activate the phosphatase activity of DUS26 independently of its AK activity (Kim et al. 2014). Therefore, we also performed dephosphorylation analysis of qGL3 on OsAK3 and the effect of OsAK3 in the dephosphorylation of qGL3 on OsGSK3, unfortunately, we did not obtain a definite result. We propose that OsAK3 interacting with qGL3 affects the interaction intensity between qGL3 and the other qGL3-interacting proteins, such as Cyclin-T1;3 for cell proliferation (Qi et al. 2012). Due to the high sequence similarity between OsAK3 and the homologous OsAK4, we also identified the interaction between OsAK4 and qGL3. The molecular mechanism of the adenylate kinases OsAK3/OsAK4 and qGL3 interactions still remain unclear.

\section{The Functions of $O s A K 3$ are Diversified}

To explore the role of OsAK3 in regulating rice growth and development, we performed RNA-seq analysis of osak3 mutant and DJ. In view of the significant enrichment of DEGs in vesicle components, we also screened some genes related to flower organ identity and development (Paul et al. 2016). We found that the expression of OsFOR1, OsMADS32, OsGRF6 and other genes were altered (Additional file 3: Table S3). Mapman analysis suggested that OsAK3 was involved in biotic and abiotic stress response (Additional file 2: Fig. S9c-e). Previous study showed that salt and submergence stress stimulates adenylate kinase activity (Samarajeewa et al. 1995). DEGs associated with stress response were listed in Additional file 3: Table S4. Based on these results, the potential molecular mechanism of OsAK3 involved in the stress response is also worth exploring.

OsAK3, as an adenylate kinase, catalyzes a reversible transphosphorylation reaction that converts ADP to ATP and AMP. Adenylate energy charge (AEC) ratio affects cellular energy status, which in turn alters energyrelated metabolic processes. GO enrichment showed that a large number of DEGs were related to lipid metabolism and carbohydrate metabolism (Fig. 7c; Additional file 2: Fig. S9a). Thus, OsAK3 may affect rice quality by regulating lipid and starch content. Terpenoids metabolic pathways were also significantly enriched in GO enrichment (Fig. 7c). Rice diterpenoids play an important role in phytohormone and phytoalexin, such as GA hormone (Wang et al. 2018). We found that GA synthesis genes (OsGA3ox1, OsGA20ox3 and OxGA20ox4) were downregulated along with down-regulated expression of GA inactivation genes (OsGA2ox1 and OsGA2ox4), which may lead to an inhibition on GA biosynthesis (Fig. 8c). Among the secondary metabolic pathways, we found 
a laccase like protein OsLAC15 associating with BR response, and previous studies reported that OsLAC15 overexpressing plants were insensitive to 24-epibrassinolide treatment (Zhang et al. 2013). Heat map and RTqPCR analysis for the expression of OsLAC15 and other laccase-related genes revealed that all these genes were up-regulated in the osak3 mutant. Thus, OsAK3 may respond to $B R$ signaling by regulating the expression of OsLACs (Additional file 2: Fig. S10d, e). Taken together, we propose a working model for the response of OsAK3 to BR signals in rice (Additional file 2: Fig. S11).

\section{Conclusions}

In this study, we found that OsAK3 regulates grain size through controlling spikelet glume cells expansion. OsAK3 participates in BR signaling response and interacts with qGL3. RNA-seq analysis revealed potential functions of OsAK3 in rice floral organ identity, stress responses and various biological processes. These results give insight into the function of adenylate kinase OsAK3 and extend our understanding of BR signaling pathway.

\section{Materials and Methods}

\section{Plant Materials and Growth Conditions}

The T-DNA insertion mutant osak3 (PFG_3A-01370.L) and osak3-r (PFG_3A-01370.R) was obtained from the Korean rice mutant library (https://signal.salk.edu). The japonica (Oryza sativa) cultivar Dongjin (DJ) was the wild-type control. To generate OsAK3-overexpression transgenic plants, the full-length coding sequence of OsAK3 was linked to the pCAMBIA-1300s plasmid to construct the 35S: OsAK3 vector. Then the vector was transformed into Agrobacterium tumefaciens (EHA105) and introduced into japonica (Oryza sativa) cultivar Nipponbare (NIP) by Agrobacterium-mediated transformation method. Rice plants were cultivated in the field under natural long days in Nanjing, China. The agronomic traits of homozygous rice plants were investigated before harvest.

\section{Total RNA Isolation and RT-qPCR Analysis}

Total RNA was extracted with a High Purity Total RNA Rapid Extraction Kit (Tiangen, Beijing, China) according to the manufacturer's instructions. First-strand cDNA was synthesized using HiScript ${ }^{\circledR}$ II Q RT SuperMix for qPCR (+gDNA wiper) Kit (Vazyme, Nanjing, China). Real-time quantitative PCR (RT-qPCR) was performed using AceQ ${ }^{\circledR}$ qPCR SYBR Green Master Mix Kit (Vazyme, Nanjing, China) and Roche 480 Real-Time PCR System following the manufacturer's instructions. The rice OsActin gene (LOC_Os03g50885) was used as an internal control and for the normalization in the analysis. The relative gene expression level was calculated using the $2^{-\Delta \Delta \mathrm{Ct}}$ method as previously reported (Gao et al. 2019). The data were presented as the mean $\pm_{S D}$ of three replicates. The primers for RT-sqPCR and RT-qPCR are listed in Additional file 3: Table S1.

\section{Yeast Two-Hybrid Assay}

The full-length coding sequence of OsAK3 and OsAK4 was cloned into pGADT7, respectively to form the prey construct AD-OsAK3 and AD-OsAK4. The respective combinations of vectors with bait construct BD-qGL3 were co-transformed into the yeast strain AH109 according to the manufacturer's instruction (Clontech, USA). The transformants were grown on synthetic defined medium (SD)/-Trp-Leu at $30{ }^{\circ} \mathrm{C}$ for 3 days and the interaction was confirmed by the colony growth in $\mathrm{SD} /$ Trp-Leu-His-Ade with 5-Bromo-4-Chloro-3-Indolyl- $\alpha$ D-Galactoside (X- $\alpha$-gal). The PCR primers used for yeast two-hybrid assay are listed in Additional file 3: Table S1.

\section{Bimolecular Fluorescence Complementation (BiFC) Assay}

For BiFC assays, OsAK3 and OsAK4 were cloned into the p2YC vector and $q G L 3$ was cloned into p2YN vector, resulting in OsAK3-YC, OsAK4-YC, qGL3 ${ }^{9311}-\mathrm{YN}$ and $\mathrm{qGL}^{\mathrm{N} 411}-\mathrm{YN}$. These recombinant plasmids and empty vectors were transformed into Agrobacterium tumefaciens strain EHA105. The corresponding Agrobacterium cells combination were injected into young leaves of Nicotiana benthamiana. The fluorescence was observed under a Zeiss LSM780 confocal microscope after growth for $36-48 \mathrm{~h}$ in darkness. The PCR primers used for BiFC assay are listed in Additional file 3: Table S1.

\section{In Vitro GST Pull-Down Assay}

To verify the in vitro interaction between OsAK3 and qGL3, the full-length coding sequence of OsAK3 was cloned into the pGEX-2T vector and transformed into the Escherichia coli strain BL21 (DE3) to express the GST-OsAK3 fusion proteins. The full-length coding sequence of $q G L 3$ was cloned into the pET-30a vector and transformed into the Escherichia coli strain BL21 (DE3) to express the His-qGL3 fusion proteins. Fusion proteins GST-OsAK3 and His-qGL3 were induced with $0.5 \mathrm{mM}$ isopropyl-b-D-thiogalactopyranoside (IPTG) at $18{ }^{\circ} \mathrm{C}$ for $12 \mathrm{~h}$. For GST pull-down assay, bacterial lysates containing GST-OsAK3 or GST were mixed with lysates containing His-qGL3. Subsequently, GST Bind Resin (Novagen) was added to the miscible liquids and incubated with the fusion proteins for $4 \mathrm{~h}$. Beads were washed three times and then boiled in $1 \times$ SDS loading buffer for $10 \mathrm{~min}$. Finally, the mixture was separated by $10 \%$ SDSPAGE. The GST antibody (Cell Signaling Technology) and His antibody (Cell Signaling Technology) were used to detect the proteins by Western blot analysis. The PCR 
primers used for GST pull-down assay are listed in Additional file 3: Table S1.

\section{Exogenous BL Treatment}

For coleoptile elongation analysis, rice seeds were grown on $0.3 \%$ agar medium with different concentrations $\mathrm{BL}$ after germination. Coleoptile lengths were measured after 5 days grown in darkness. For lamina inclination assays, segments of the second leaf blade, lamina joint and $1 \mathrm{~cm}$ of leaf sheath were cut off from 1-week-old dark grown rice and inserted vertically into the $0.3 \%$ agar medium with different concentrations BL. The angles of lamina joint bending were measured after $72 \mathrm{~h}$ grown in dark. For root inhibition analysis, rice seeds were sowed and grown in the solution culture with different concentrations BL after germination. Root lengths were measured after 5 days grown in darkness. For OsAK3 transcript measurement, 1-week-old DJ seedlings were treated with $10^{-6} \mathrm{M} \mathrm{BL}$ and sampled at $0,0.5,1,2,3$ and $6 \mathrm{~h}$.

\section{Subcellular Localization}

To determine the subcellular localization of OsAK3, the full-length OsAK3 coding sequence was fused with green fluorescent protein (GFP) in the pAN580 vector to produce the OsAK3-GFP fusion protein in plants. Rice protoplasts were isolated from 2-week-old rice seedlings and transfected with $10 \mu \mathrm{g}$ plasmid DNA by PEG-mediated transformation methods. The GFP signal was visualized using a confocal laser scanning microscope (LSM780, Zeiss, Germany) after incubation at $26{ }^{\circ} \mathrm{C}$ for $12 \mathrm{~h}$. The PCR primers used for transient expression assay are listed in Additional file 3: Table S1.

\section{Scanning Electron Microscopy Observation of Spikelet Hull} Mature rice seeds of WT and transgenic lines were collected and were fixed in FAA solution, dehydrated in series concentrations of ethanol, and critical point-dried in a vacuum freeze drier. Subsequently, samples were mounted, coated with gold, and finally observed under a scanning electron microscopy (Hitachi, Japan). The cell size of each sample was measured using ImageJ software.

\section{RNA-Seq Analysis}

Young panicles of WT and osak3 mutant were sampled for RNA-seq analysis with three biological replicates. The extraction and examination of total RNA, library construction and Illumina sequencing were done by Personal Biotech (Shanghai, China) using the Illumina novaseq pe150. The clean reads were aligned with reference sequences of rice in IRGSP-1.0 (http://rapdb.dna.affrc.go. jp/download/irgsp1.html). Differentially expressed genes (DEGs) were defined by absolute $\log _{2}$ Fold Change $>1$ and $P$ value $<0.05$. The DEGs were classified according to Gene Ontology (GO) annotation using AgriGO (http:// bioinfo.cau.edu.cn/agriGO). The selected differential expressed genes were blasted using the RGAP database (http://rice.plantbiology.msu.edu/) and the NCBI database (https://www.ncbi.nlm.nih.gov/).

\section{Phylogenetic Analysis}

The sequences of rice, Arabidopsis and human adenylate kinases were downloaded from NCBI (https://www.ncbi. nlm.nih.gov/). Multiple sequence alignments of these homologs were performed using Muscle (MEGA6) and the phylogenetic tree was constructed using the Neighbor-Joining method (MEGA6). Bootstrap values were obtained by 1,000 bootstrap replicates. The amino acid sequences used for phylogenetic analysis are listed in Additional file 1: Supplemental Data Set 1.

\begin{abstract}
Abbreviations
BRs: Brassinosteroids; BL: Brassinolide; CS: Castasterone; GIP: qGL3-interacting protein; TF: Transcription factor; AK: Adenylate kinase; ATP: Adenosine triphosphate; ADP: Adenosine diphosphate; DJ: Dongjin; NIP: Nipponbare; PADK1: PLASTIDIAL ADENYLATE KINASE 1; AMK2: ADENOSINE MONOPHOSPHATE KINASE 2; Y2H:Yeast two-hybrid; RT-sqPCR: Reverse transcription semiquantitative PCR; RT-qPCR: Quantitative real-time PCR; GFP: Green fluorescent protein; GST: Glutathione S-transferase; BiFC: Bimolecular fluorescence complementation; YFP: Yellow fluorescent protein; SEM: Scanning electron microscopy; DEG: Differentially expressed gene; GO: Gene Ontology; BP: Biological process; MF: Molecular function; CC: Cell component; AEC: Adenylate energy charge.
\end{abstract}

\section{Supplementary Information}

The online version contains supplementary material available at https://doi. org/10.1186/s12284-021-00546-0.

Additional file 1: Supplemental Data Set 1. Adenylate kinase sequences used in phylogenetic tree analysis.

Additional file 2: Fig. S1. Colorimetric quantitation of OsAK3 activity in the presence of qGL3 in vitro. Fig. S2. OsAK4 has the highest homology with OSAK3 and interacts with qGL3. Fig. S3. Genotyping analysis of osak3 and osak3-r mutant. Fig. S4. Morphological characteristics of osak3-r mutant. Fig. S5. Effects of BL treatment in osak3-r. Fig. S6. Effects of BL treatment in osak3. Fig. S7. Effects of BL treatment in OsAK3-OXs plants. Fig. S8. RT-qPCR verification of DEGs selected from RNA-seq. Fig. S9. Overview of the DEGs between DJ and osak3 mutant. Fig. S10. OsAK3 is involved in multiple phytohormone signaling pathways and stress responses. Fig. S11. A working model for the functions of OsAK3 in BR signaling and plant growth and development.

Additional file 3: Table S1. Primers used in this study. Table S2. List of DEGs used for RT-qPCR validation. Table S3. List of DEGs associated with floral organ development detected in DJ and osak3. Table S4. List of DEGs associated with stress response detected in DJ and osak3.

\section{Acknowledgements}

We would like to thank the colleagues at the Jiangsu Collaborative Innovation Center for Modern Crop Production, Cyrus Tang Seed Innovation Center, and Nanjing Agricultural University for reading and participating in discussions relating to the preparation of this manuscript. 


\section{Authors' Contributions}

J.Z., X.G., and J.H. designed the research; J.Z. performed most of the research; G.C., Y.W., J.L., H.D., and R.W. helped perform some experiments; J.Z., X.G., and J.H. wrote the paper; H.Z. and J.H. supervised this research. All authors read and approved of the manuscript.

\section{Funding}

This work was supported by the Youth Science Foundation of Jiangsu Province (SBK2019040714), Natural Science Foundation of China (32071918, 32000227) and the Fundamental Research Funds for the Central Universities (KJQN202102).

\section{Availability of Data and Materials}

The datasets supporting the conclusions of this article are included within the article and its additional files.

\section{Declarations}

\section{Ethics Approval and Consent to Participate}

Not applicable.

\section{Consent for Publication}

Not applicable.

\section{Competing Interests}

The authors declare that there is no conflict of interest.

\section{Author details}

${ }^{1}$ State Key Laboratory of Crop Genetics and Germplasm Enhancement, College of Agriculture, Nanjing Agricultural University, Nanjing 210095, China. ${ }^{2}$ Jiangsu Provincial Engineering Research Center of Seed Industry Science and Technology, Nanjing 210095, China.

Received: 7 July 2021 Accepted: 17 December 2021

Published online: 28 December 2021

\section{References}

Amiri M, Conserva F, Panayiotou C, Karlsson A, Solaroli N (2013) The human adenylate kinase 9 is a nucleoside mono- and diphosphate kinase. Int J Biochem Cell Biol 45:925-931

Aya K, Hobo T, Sato-Izawa K, Ueguchi-Tanaka M, Kitano H, Matsuoka M (2014) A novel AP2-type transcription factor, SMALL ORGAN SIZE1, controls organ size downstream of an auxin signaling pathway. Plant Cell Physiol 55:897-912

Carrari F, Coll-Garcia D, Schauer N, Lytovchenko A, Palacios-Rojas N, Balbo I, Rosso M, Fernie AR (2005) Deficiency of a plastidial adenylate kinase in Arabidopsis results in elevated photosynthetic amino acid biosynthesis and enhanced growth. Plant Physiol 137:70-82

Che R, Tong H, Shi B, Liu Y, Fang S, Liu D, Xiao Y, Hu B, Liu L, Wang H, Zhao M, Chu C (2015) Control of grain size and rice yield by GL2-mediated brassinosteroid responses. Nat Plants 2:15195

Clouse SD, Sasse JM (1998) BRASSINOSTEROIDS: essential regulators of plant growth and development. Annu Rev Plant Physiol Plant Mol Biol 49:427-451

Dzeja P, Terzic A (2009) Adenylate kinase and AMP signaling networks: metabolic monitoring, signal communication and body energy sensing. Int J Mol Sci 10:1729-1772

Fan C, Xing Y, Mao H, Lu T, Han B, Xu C, Li X, Zhang Q (2006) GS3, a major QTL for grain length and weight and minor QTL for grain width and thickness in rice, encodes a putative transmembrane protein. Theor Appl Genet 112:1164-1171

Feng X, Yang R, Zheng X, Zhang F (2012) Identification of a novel nuclearlocalized adenylate kinase 6 from Arabidopsis thaliana as an essential stem growth factor. Plant Physiol Biochem 61:180-186

Feng Z, Wu C, Wang C, Roh J, Zhang L, Chen J, Zhang S, Zhang H, Yang C, Hu J, You X, Liu X, Yang X, Guo X, Zhang X, Wu F, Terzaghi W, Kim SK, Jiang L, Wan J (2016) SLG controls grain size and leaf angle by modulating brassinosteroid homeostasis in rice. J Exp Bot 67:4241-4253
Gachon CM, Langlois-Meurinne M, Saindrenan P (2005) Plant secondary metabolism glycosyltransferases: the emerging functional analysis. Trends Plant Sci 10:542-549

Gao X, Zhang JQ, Zhang X, Zhou J, Jiang Z, Huang P, Tang Z, Bao Y, Cheng J, Tang H, Zhang W, Zhang H, Huang J (2019) Rice qGL3/OsPPKL1 functions with the GSK3/SHAGGY-like kinase OsGSK3 to modulate brassinosteroid signaling. Plant Cell 31:1077-1093

Harberd NP (2015) Shaping taste: the molecular discovery of rice genes improving grain size, shape and quality. J Genet Genom 42:597-599

Huang K, Wang D, Duan P, Zhang B, Xu R, Li N, Li Y (2017) WIDE AND THICK GRAIN 1, which encodes an otubain-like protease with deubiquitination activity, influences grain size and shape in rice. Plant J 91:849-860

Ionescu MI (2019) Adenylate kinase: a ubiquitous enzyme correlated with medical conditions. Protein J 38:120-133

Kawai M, Kidou S, Kato A, Uchimiya H (1992) Molecular characterization of cDNA encoding for adenylate kinase of rice (Oryza sativa L.). Plant J 2:845-854

Kim H, Lee HJ, Oh Y, Choi SG, Hong SH, Kim HJ, Lee SY, Choi JW, Su Hwang D, Kim KS, Kim HJ, Zhang J, Youn HJ, Noh DY, Jung YK (2014) The DUSP26 phosphatase activator adenylate kinase 2 regulates FADD phosphorylation and cell growth. Nat Commun 5:3351

Lange PR, Geserick C, Tischendorf G, Zrenner R (2008) Functions of chloroplastic adenylate kinases in Arabidopsis. Plant Physiol 146:492-504

Li D, Wang L, Wang M, Xu YY, Luo W, Liu YJ, Xu ZH, Li J, Chong K (2009) Engineering OsBAK1 gene as a molecular tool to improve rice architecture for high yield. Plant Biotechnol J 7:791-806

Li N, Xu R, Duan P, Li Y (2018) Control of grain size in rice. Plant Reprod 31:237-251

Li JG, Fan M, Hua W, Tian Y, Chen LG, Sun Y, Bai MY (2020) Brassinosteroid and hydrogen peroxide interdependently induce stomatal opening by promoting guard cell starch degradation. Plant Cell 32:984-999

Liu L, Tong H, Xiao Y, Che R, Xu F, Hu B, Liang C, Chu J, Li J, Chu C (2015a) Activation of Big Grain 1 significantly improves grain size by regulating auxin transport in rice. Proc Natl Acad Sci USA 112:11102-11107

Liu S, Hua L, Dong S, Chen H, Zhu X, Jiang J, Zhang F, Li Y, Fang X, Chen F (2015b) OsMAPK6, a mitogen-activated protein kinase, influences rice grain size and biomass production. Plant J 84:672-681

Noma T, Fujisawa K, Yamashiro Y, Shinohara M, Nakazawa A, Gondo T, Ishihara T, Yoshinobu K (2001) Structure and expression of human mitochondrial adenylate kinase targeted to the mitochondrial matrix. Biochem J 358:225-232

Panayiotou C, Solaroli N, Xu Y, Johansson M, Karlsson A (2011) The characterization of human adenylate kinases 7 and 8 demonstrates differences in kinetic parameters and structural organization among the family of adenylate kinase isoenzymes. Biochem J 433:527-534

Panayiotou C, Solaroli N, Karlsson A (2014) The many isoforms of human adenylate kinases. Int J Biochem Cell Biol 49:75-83

Paul P, Röth S, Schleiff E (2016) Importance of organellar proteins, protein translocation and vesicle transport routes for pollen development and function. Plant Reprod 29:53-65

Qi P, Lin YS, Song XJ, Shen JB, Huang W, Shan JX, Zhu MZ, Jiang L, Gao JP, Lin HX (2012) The novel quantitative trait locus GL3.1 controls rice grain size and yield by regulating Cyclin-T1;3. Cell Res 22:1666-1680

Qiao S, Sun S, Wang L, Wu Z, Li C, Li X, Wang T, Leng L, Tian W, Lu T, Wang $X$ (2017) The RLA1/SMOS1 transcription factor functions with OsBZR1 to regulate brassinosteroid signaling and rice architecture. Plant Cell 29:292-309

Ren H, Wang L, Bennett M, Liang Y, Zheng X, Lu F, Li L, Nan J, Luo M, Eriksson S, Zhang C, Su XD (2005) The crystal structure of human adenylate kinase 6: an adenylate kinase localized to the cell nucleus. Proc Natl Acad Sci USA 102:303-308

Samarajeewa PK, Kawai M, Anai T, Hirai A, Uchimiya H (1995) Sodium chloride stimulates adenylate kinase level in seedlings of salt-sensitive rice varieties. J Plant Physiol 147:277-280

Slovak R, Setzer C, Roiuk M, Bertels J, Göschl C, Jandrasits K, Beemster GTS, Busch W (2020) Ribosome assembly factor Adenylate Kinase 6 maintains cell proliferation and cell size homeostasis during root growth. New Phytol 225:2064-2076

Song XJ, Huang W, Shi M, Zhu MZ, Lin HX (2007) A QTL for rice grain width and weight encodes a previously unknown RING-type E3 ubiquitin ligase. Nat Genet 39:623-630 
Sun S, Wang L, Mao H, Shao L, Li X, Xiao J, Ouyang Y, Zhang Q (2018) A G-protein pathway determines grain size in rice. Nat Commun 9:851

Tong H, Chu C (2018) Functional specificities of brassinosteroid and potential utilization for crop improvement. Trends Plant Sci 23:1016-1028

Tong H, Liu L, Jin Y, Du L, Yin Y, Qian Q, Zhu L, Chu C (2012) DWARF AND LOW-TILLERING acts as a direct downstream target of a GSK3/SHAGGYlike kinase to mediate brassinosteroid responses in rice. Plant Cell 24:2562-2577

Utsunomiya Y, Samejima C, Takayanagi Y, Izawa Y, Yoshida T, Sawada Y, Fujisawa Y, Kato H, Iwasaki Y (2011) Suppression of the rice heterotrimeric $G$ protein $\beta$-subunit gene, RGB1, causes dwarfism and browning of internodes and lamina joint regions. Plant J 67:907-916

Wang M, Lu X, Xu G, Yin X, Cui Y, Huang L, Rocha P, Xia X (2016) OsSGL, a novel pleiotropic stress-related gene enhances grain length and yield in rice. Sci Rep 6:38157

Wang W, Li Y, Dang P, Zhao S, Lai D, Zhou L (2018) Rice secondary metabolites: structures, roles, biosynthesis, and metabolic regulation. Molecules 23:3098

Wei X, Song X, Wei L, Tang S, Sun J, Hu P, Cao X (2017) An epiallele of rice AK1 affects photosynthetic capacity. J Integr Plant Biol 59:158-163

Xiao Y, Liu D, Zhang G, Tong H, Chu C (2017) Brassinosteroids regulate OFP1, a DLT interacting protein, to modulate plant architecture and grain morphology in rice. Front Plant Sci 8:1698

Xiao Y, Liu D, Zhang G, Gao S, Liu L, Xu F, Che R, Wang Y, Tong H, Chu C (2019) Big Grain3, encoding a purine permease, regulates grain size via modulating cytokinin transport in rice. J Integr Plant Biol 61:581-597

Xiao Y, Zhang G, Liu D, Niu M, Tong H, Chu C (2020) GSK2 stabilizes OFP3 to suppress brassinosteroid responses in rice. Plant J 102:1187-1201

Xu C, Liu Y, Li Y, Xu X, Xu C, Li X, Xiao J, Zhang Q (2015) Differential expression of GS5 regulates grain size in rice. J Exp Bot 66:2611-2623

Xu R, Duan P, Yu H, Zhou Z, Zhang B, Wang R, Li J, Zhang G, Zhuang S, Lyu J, Li N, Chai T, Tian Z, Yao S, Li Y (2018) Control of grain size and weight by the OsMKKK10-OsMKK4-OsMAPK6 signaling pathway in rice. Mol Plant $11: 860-873$

Yamamuro C, Ihara Y, Wu X, Noguchi T, Fujioka S, Takatsuto S, Ashikari M, Kitano $H$, Matsuoka M (2000) Loss of function of a rice brassinosteroid insensitive1 homolog prevents internode elongation and bending of the lamina joint. Plant Cell 12:1591-1606

Yang C, Shen W, He Y, Tian Z, Li J (2016) OVATE family protein 8 positively mediates brassinosteroid signaling through interacting with the GSK3-like kinase in rice. PLoS Genet 12:e1006118

Yang C, Ma Y, He Y, Tian Z, Li J (2018) OsOFP19 modulates plant architecture by integrating the cell division pattern and brassinosteroid signaling. Plant J 93:489-501

Zhang LY, Bai MY, Wu J, Zhu JY, Wang H, Zhang Z, Wang W, Sun Y, Zhao J, Sun X, Yang H, Xu Y, Kim SH, Fujioka S, Lin WH, Chong K, Lu T, Wang ZY (2009) Antagonistic $\mathrm{HLH} / \mathrm{bHLH}$ transcription factors mediate brassinosteroid regulation of cell elongation and plant development in rice and Arabidopsis. Plant Cell 21:3767-3780

Zhang C, Xu Y, Guo S, Zhu J, Huan Q, Liu H, Wang L, Luo G, Wang X, Chong K (2012a) Dynamics of brassinosteroid response modulated by negative regulator LIC in rice. PLoS Genet 8:e1002686

Zhang X, Wang J, Huang J, Lan H, Wang C, Yin C, Wu Y, Tang H, Qian Q, Li J, Zhang H (2012b) Rare allele of OsPPKL1 associated with grain length causes extra-large grain and a significant yield increase in rice. Proc Natl Acad Sci USA 109:21534-21539

Zhang YC, Yu Y, Wang CY, Li ZY, Liu Q, Xu J, Liao JY, Wang XJ, Qu LH, Chen F, Xin P, Yan C, Chu J, Li HQ, Chen YQ (2013) Overexpression of microRNA OsmiR397 improves rice yield by increasing grain size and promoting panicle branching. Nat Biotechnol 31:848-852

Zhang B, Wang X, Zhao Z, Wang R, Huang X, Zhu Y, Yuan L, Wang Y, Xu X, Burlingame AL, Gao Y, Sun Y, Tang W (2016) OsBRI1 activates BR signaling by preventing binding between the TPR and kinase domains of OsBSK3 via phosphorylation. Plant Physiol 170:1149-1161

Zhang Y, Launay H, Liu F, Lebrun R, Gontero B (2018a) Interaction between adenylate kinase 3 and glyceraldehyde-3-phosphate dehydrogenase from Chlamydomonas reinhardtii. FEBS J 285:2495-2503

Zhang Z, Li J, Tang Z, Sun X, Zhang H, Yu J, Yao G, Li G, Guo H, Li J, Wu H, Huang H, Xu Y, Yin Z, Qi Y, Huang R, Yang W, Li Z (2018b) Gnp4/LAX2, a RAWUL protein, interferes with the OSIAA3-OSARF25 interaction to regulate grain length via the auxin signaling pathway in rice. J Exp Bot 69:4723-4737
Zuo J, Li J (2014) Molecular genetic dissection of quantitative trait loci regulating rice grain size. Annu Rev Genet 48:99-118

\section{Publisher's Note}

Springer Nature remains neutral with regard to jurisdictional claims in published maps and institutional affiliations.

\section{Submit your manuscript to a SpringerOpen ${ }^{\circ}$ journal and benefit from:}

- Convenient online submission

- Rigorous peer review

- Open access: articles freely available online

- High visibility within the field

- Retaining the copyright to your article

Submit your next manuscript at $\boldsymbol{\nabla}$ springeropen.com 